\title{
AN INFORMATION-THEORETIC IMAGE QUALITY MEASURE: COMPARISON WITH STATISTICAL SIMILARITY
}

\author{
${ }^{1}$ Asmhan Flieh Hassan, ${ }^{2}$ Dong Cai-lin and ${ }^{3}$ Zahir M. Hussain \\ ${ }^{1} \mathrm{PhD}$ Candidate, School of Mathematics and Statistics, HuaZhong Normal University, \\ 152 Luoyu Road, Wuhan, 430079, China \\ Department of Mathematics University of Kufa, Najaf, Iraq \\ ${ }^{2}$ School of Mathematics and Statistics, HuaZhong Normal University, \\ 152 Luoyu Road, Wuhan, 430079, China \\ ${ }^{3}$ Department of Computer Science, University of Kufa, Najaf, Iraq, \\ School of Engineering, Edith Cowan University, Australia
}

Received 2014-05-02; Revised 2014-09-07; Accepted 2014-11-28

Funding: Ministry of Higher Education and Scientific Research (Iraq), HuaZhong Normal University (China).

Competing Interests: The authors have declared that no competing interests exist

\begin{abstract}
We present an information-theoretic approach for structural similarity for assessing gray scale image quality. The structural similarity measure SSIM, proposed in 2004, has been successflly used and verfied. SSIM is based on statistical similarity between the two images. However, SSIM can produce confusing results in some cases where it may give a non-trivial amount of similarity for two different images. Also, SSIM cannot perform well (in detecting similarity or dissimilarity) at low peak signal to noise ratio (PSNR). In this study, we present a novel image similarity measure, HSSIM, by using information - theoretic technique based on joint histogram. The proposed method has been tested under Gaussian noise. Simulation results show that the proposed measure HSSIM outperforms statistical similarity SSIM by ability to detect similarity under very low PSNR. The average difference is about $20 \mathrm{~dB}$.
\end{abstract}

Keywords: Joint Histogram, Image Structural Similarity, Image Quality Assessment, Image Processing

\section{INTRODUCTION}

Image similarity measurement is a fundamental issue in real-world applications. Image quality metrics play a significant role in image processing. It can be used to adjust image quality and optimize parameters in many image processing applications such as enhancement, compression, restoration, etc. The image quality assessment aims to design methods for objective assessment of quality versus subjective human image quality evaluation (Wang et al., 2002).

A simple way to measure the similarity between two images is to calculate the Mean-Squared Error (MSE) between the reference image and the distorted version. MSE is easy to compute, however, it performs poorly in pattern recognition (Wang et al., 2004; Simard et al., 2002).

An important objective measure was proposed in 2004 by (Wang and Bovik, 2004), where image distortion was considerd as a combination of three kinds of distortion: Correlation, luminance and contrast.

In general, image similarity measures can be classsifed into two main directions: Statistical-based and information-theoretic based quality measures.

\subsection{Statistical Measures}

The above measure proposed by Wang et al. (2004) which was called SSIM, used distance Corresponding Author: Hussain, Z.M., Department of Computer Science, University of Kufa, P.O. Box 21, Kufa, Najaf, Iraq 
covariance to measure the structural similarity based on statistical measurements such as mean and standard deviation as follows Equation 1:

$$
\rho(x, y)=\frac{\left(2 \mu_{x} \mu_{y+} C_{1}\right)\left(2 \sigma_{x y}+C_{2}\right)}{\left(\mu_{x}^{2}+\mu_{y}^{2}+C_{1}\right)\left(\sigma_{x}^{2}+\sigma_{y}^{2}+C_{2}\right)}
$$

where, $\rho(x, y)$ is the structural SSIM measure between the image $\mathrm{x}$ (original or reference image) and the image $\mathrm{y}$ (corrupted image), $\mu_{x}, \mu_{y}, \sigma_{x}^{2}$ and $\sigma_{y}^{2}$ are the statistical means and variances of pixel values in images $\mathrm{x}$ and $\mathrm{y}$, $\sigma_{x y}$ is the statistical covariance between images $\mathrm{x}$ and $\mathrm{y}$, while the constants $\mathrm{C}_{1}$ and $\mathrm{C}_{2}$ are given by $\mathrm{C}_{1}=\left(\mathrm{K}_{1} \mathrm{~L}\right)^{2}$ and $\mathrm{C}_{2}=\left(\mathrm{K}_{2} \mathrm{~L}\right)^{2}$, with $\mathrm{K}_{1}$ and $\mathrm{K}_{2}$ are small constants and $\mathrm{L}=255$ (the maximum pixel value).

Sheikh et al. (2006), proposed further study on quality assessment. In Sampat et al. (2009) an improvement of SSIM was presented based on wavelet coefficients which are extracted at the same locations in the same wavelet sub bands of the two images; and the new measure proved to be insensitive to small geometric variations.

Some limitations of Image Quality Measurement are presented in (Zhang et al., 2009).

SSIM gives high level of similarity for noise free condition while it fails when noise increases. In addition, if the two images are dissimilar, SSIM may give an amount of similarity (Hashim and Hussain, 2014). This is so because SSIM can't reveal all image structural properties, so we need to more specific measurements that are image-dependent. Hashim and Hussain (2014) proposed methods to determine a reliable similarity between any two images, similar or dissimilar. The methods are based on image-dependent properties, specifically the results of edge detection and segmentation methods, in addition to the image statistical properties; and tested under effects of Gaussian noise, impulse noise, as well as blur.

The above-mentioned similarity measures are all analysed based on statistical moments, which we will study in this study versus information-theoretic approach in order to test similarity.

\subsection{Information-Theoretic Measures}

The use of information-theoretic analysis in image processing is possible if we assume that images are $2 \mathrm{D}$ random variables.

The most common measure of information is the Shannon-Wiener entropy measure. The entropy $\mathrm{H}$ of a discrete random variable $X$ with the values in the set $\left\{x_{1}\right.$, $\left.\mathrm{x}_{2}, \ldots, \mathrm{x}_{\mathrm{n}}\right\}$ is defined as (Shannon and Weaver, 1967):

$$
H(X)=-\sum_{i=1}^{n} p_{i} \log p_{i}
$$

Where:

$$
p_{\mathrm{i}}=p_{\mathrm{r}}\left[X=x_{i}\right]
$$

The definition of entropy for a single random variable can be extended to a pair of random variables.

The joint entropy $\mathrm{H}(\mathrm{X}, \mathrm{Y})$ of a pair of discrete $2 \mathrm{D}$ random variables, which are images in our case, with a joint distribution $\mathrm{p}_{\mathrm{ij}}$ is given by (Viola and Wells, 1995):

$$
H(X, Y)=-\sum_{\mathrm{i}=1}^{\mathrm{n}} \sum_{\mathrm{j}=1}^{\mathrm{m}} p_{\mathrm{ij}} \log p_{\mathrm{ij}}
$$

The mutual information between two discrete random variables $\mathrm{X}$ and $\mathrm{Y}$ is defined as (Viola and Wells, 1995):

$$
I(X, Y)=-\sum_{\mathrm{i}=1}^{\mathrm{n}} \sum_{\mathrm{j}=1}^{\mathrm{m}} p_{\mathrm{ij}} \log \frac{p_{\mathrm{ij}}}{p_{\mathrm{i}} p_{\mathrm{j}}}
$$

The mutual information between two random variables can be defined as the amount of information that one random variable can give about the other one. Hence, mutual information as defined above is a measure of the decrease in the entropy of one of these variables if we are given the other one. As such, it is possible to use mutual information as well as other information-theoretic measures (like the histogram) to define similarity measures.

The joint intensity histogram for two images would be spread (hence, giving high joint entropy) when the two images are not aligned, while it is compact (hence, giving low joint entropy) when the two images are aligned. The Mutual Information (MI) is proposed by various authors as per (Wells et al., 1996; Maes et al., 1997; Pluim et al., 2003). The overlap invariant Normalized MI (NMI) have been proposed in (Studholme et al., 1999).

Regional MI (RMI) has been proposed in (Studholme et al., 2006), while Conditional MI (CMI) has been proposed in (Loeckx et al., 2010). These methods depend on summing local MI for regions of the images, rather than finding the global MI. Klein et al. 
(2007; 2009) proposed Localized MI (LMI); where random samples are taken from regions in very iteration.

Zhuang et al. (2011) proposed spatially-encoded MI, where pixels are assigned weights based on their spatial location instead of having equal weights.

Darkner and Sporring (2013) has proposed a unified approach for NMI.

In this study, we present an information-theoretic image similarity measure and show its superior performance versus the classical similarity measure SSIM under additive Gaussian noise with several ratios of signal to noise.

\section{JOINT HISTOGRAM}

The concept of multidimensional histogram has been investigated by several researchers in different application domains (Hill et al., 1993; Pass and Zabih, 1999).

The joint-histogram represents the joint-probability of two or more random variables, images in our case. For the two-dimensional joint-histogram, the pixel values of two images are used as the coordinate axis. Given two images $\mathrm{A}$ and $\mathrm{B}$ and pixel intensity values $i$ and $j, \mathrm{H}_{\mathrm{ij}}$ (A, B) represents the probability of a pixel to have the value $i$ on the image $A$ and $j$ on the image $B$. Hence, each entry is the number of times an intensity $i$ in one image corresponds to an intensity $j$ in the other.

If the joint histogram is normalized, it becomes an estimate of the joint Probability Distribution Function (PDF) of intensities in the images (Kuczynski and Miko£Ajczak, 2003).

We can notice that under noise-free conditions, the joint histogram of two identical images will give a straight-line peak at $\mathrm{i}=\mathrm{j}$, while under noisy conditions this criterion is perturbed. Based on this approach, we designed a novel similarity measure as follows.

\section{RATIONALE}

We noticed that SSIM measure introduced in (Wang et al., 2004) gives good measure of similarity between two similar images; however, it fails when noise is significant (i.e., at low PSNR). We need an enhanced measure that can perform well under low PSNR. We have utilized joint histogram and combined it with the original histogram to get the enhanced measure HSSIM; also we tested SSIM and HSSIM under disruptive conditions like additive Gaussian noise.

\section{THE PROPOSED IT MEASURE}

The original design of structural similarity measure SSIM was based on image statistical properties as in
(Wang et al., 2004). In this paper we focus on image information-theoretic properties, specifically the joint histogram and propose the following image-dependent measure. First, we propose an error estimate between an original (reference) image $\mathrm{x}$ and a noisy version $\mathrm{y}$ of it. The estimate is based on the diagonal symmetry of selfjoint histogram $\mathrm{H}$, which is just a $2 \mathrm{D}$ extension of the reference image histogram:

$$
h(x)=\left\{h_{i}(x), i \in(1,2, \cdots, N)\right\}
$$

about $i=j$ axis as follows:

$$
H_{i j}(x, x)=H_{j i}(x, x)
$$

The Information-Theoretic (IT) error estimate of joint histograms (relative to the original histogram) can be designed as follows Equation 2:

$$
E(x, y)=\sqrt{\frac{\sum_{i} \sum_{j}\left[\left(H_{i j}-H_{j i}\right) \frac{1}{h_{i}+c}\right]^{2}}{2 L^{2}}} \ldots
$$

where, $\mathrm{c}$ is a very small positive constant, inserted mainly to avoid division by zero. Note that:

$$
E(x, y) \geq 0
$$

A maximal error estimate $\mathrm{E} \infty(\mathrm{x}, \mathrm{y})$ can be considered when noise power is very high (very low PSNR). Then the above estimate can be normalized with respect to the maximal relative error estimate as follows Equation 3:

$e(x, y)=E(x, y) / E_{\infty}(x, y)$

The normalization process will ensure that:

$0 \leq e(x, y) \leq 1$

Based on the above error estimate an informationtheoretic similarity measure (which we name as HSSIM) can be proposed as follows Equation 5 and 6:

$\lambda(x, y)=1-e(x, y)$

where:

$0 \leq \lambda(x, y) \leq 1$ 
As in the case of SSIM, HSSIM ranges between 0 (for dissimilar images) and 1 (for identical images). If we denote SSIM by $\rho(x, y)$ then we have the i equality:

$$
0 \leq \lambda(x, y) \leq 1
$$

where, $\rho(x, y)=1$ for identical images, while $\rho(x, y)=0$ for totally different images.

\section{THE TEST ENVIRONMENT}

The proposed HSSIM measure have been tested under Gaussian noise, which is the main source of noise in many image processing systems.

Different kinds of images has been used: A human face, a landscape and a geometric shape.

\section{RESULTS AND DISCUSSION}

The above algorithms have been simulated using MATLAB. Figure 1 to 3 show performance of SSIM and HSSIM using similar images under Gaussian noise. Note that:

$$
0 \leq \lambda(x, y), \rho(x, y) \leq 1
$$

For completely similar images we have:

$$
\lambda(x, y)=\rho(x, y)=1
$$

while for totally different images we have:

$$
\lambda(x, y)=\rho(x, y)=0
$$

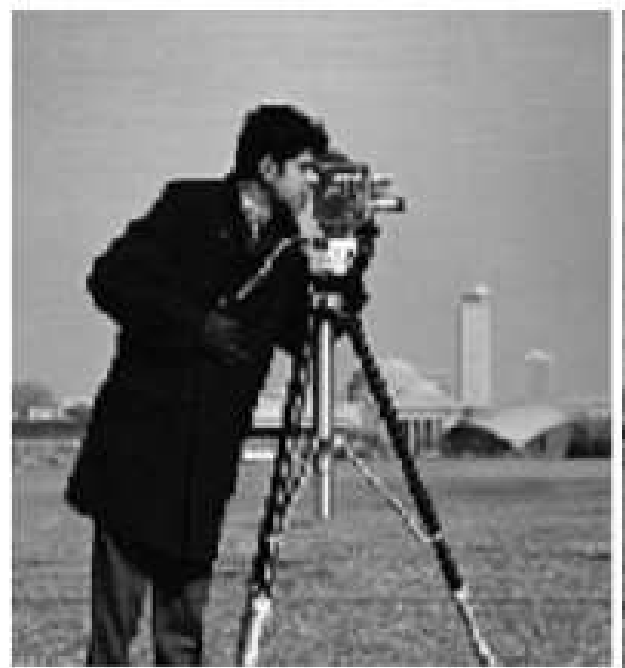

To calculate similarity measure SSIM, an $\mathrm{M} \times \mathrm{M}$ window $(\mathrm{M}=11)$ is used with a standard deviation of 1.5 (Wang et al., 2004). The constants $\mathrm{C}_{1}=\left(\mathrm{K}_{1} \mathrm{~L}\right)^{2}$ and $\mathrm{C}_{1}=\left(\mathrm{K}_{2} \mathrm{~L}\right)^{2}\left(\mathrm{~K}_{1}\right.$ and $\mathrm{K}_{2}$ being small constants, $\mathrm{L}=$ 255) where chosen as in (Wang et al., 2004) as $\mathrm{K}_{1}=$ 0.01 and $\mathrm{K}_{2}=0.03$. Note that the performance of SSIM is insensitive to these constants (Wang et al., 2004; Wang and Bovik, 2004). The constant in Equation 1 is taken as $\mathrm{c}=1 \times 10^{-15}$.

We also implemented the joint Histogram-based measure (HSSIM) as per Equation 4. $\mathrm{E} \infty(\mathrm{x}, \mathrm{y})$ is calculated at PSNR $=-100 \mathrm{~dB}$ (total noise).

\subsection{Performance under Gaussian Noise}

After we implemented the joint histogram-based measure (HSSIM) as per Equation 4 and SSIM as per Equation 1, we tested their performance of detecting similarity under noisy conditions, specifically when the other image is corrupted with Gaussian noise. Peak signal to noise ratio (PSNR) was used in this test as follows:

$$
P S N R=\frac{L^{2}}{p_{n}}
$$

where, $p_{n}$ is the Gaussian noise variance (power).

A maximal error estimate can be considered when noise power is very high with PSNR $=100 \mathrm{~dB}$.

The result of using two similar images (a reference image and a noisy version of it) is shown in Fig. 1 to 3 for various kinds of images: Landscape, human face and geometric shape.

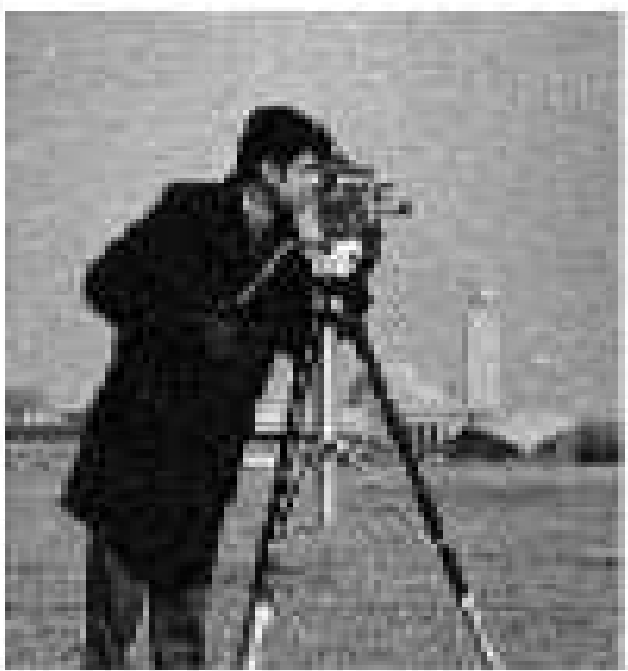

(a) 
Asmhan Flieh Hassan et al. / Journal of Computer Science 10 (11): 2269.2283, 2014
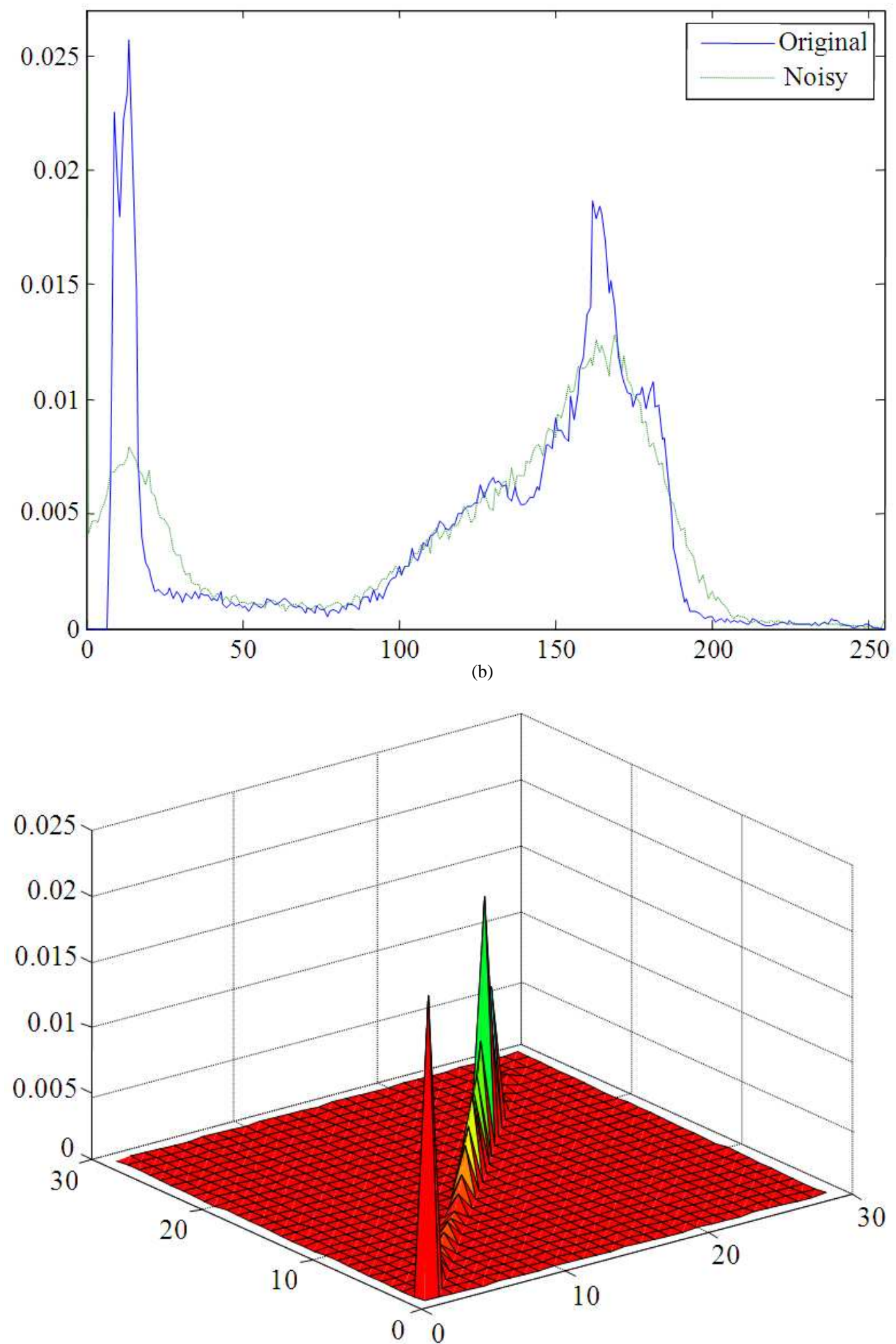

(c) 
Asmhan Flieh Hassan et al. / Journal of Computer Science 10 (11): 2269.2283, 2014

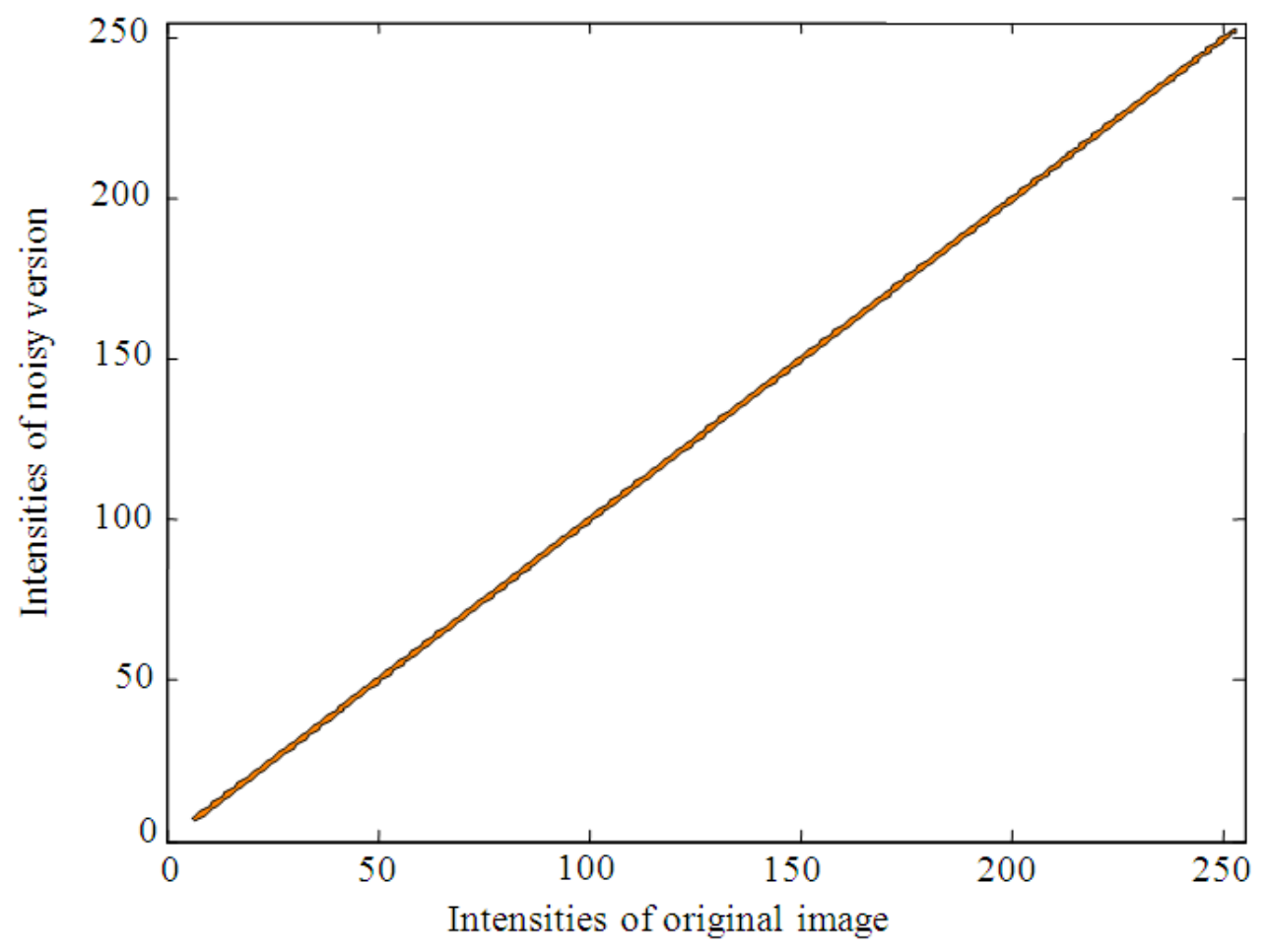

(d)

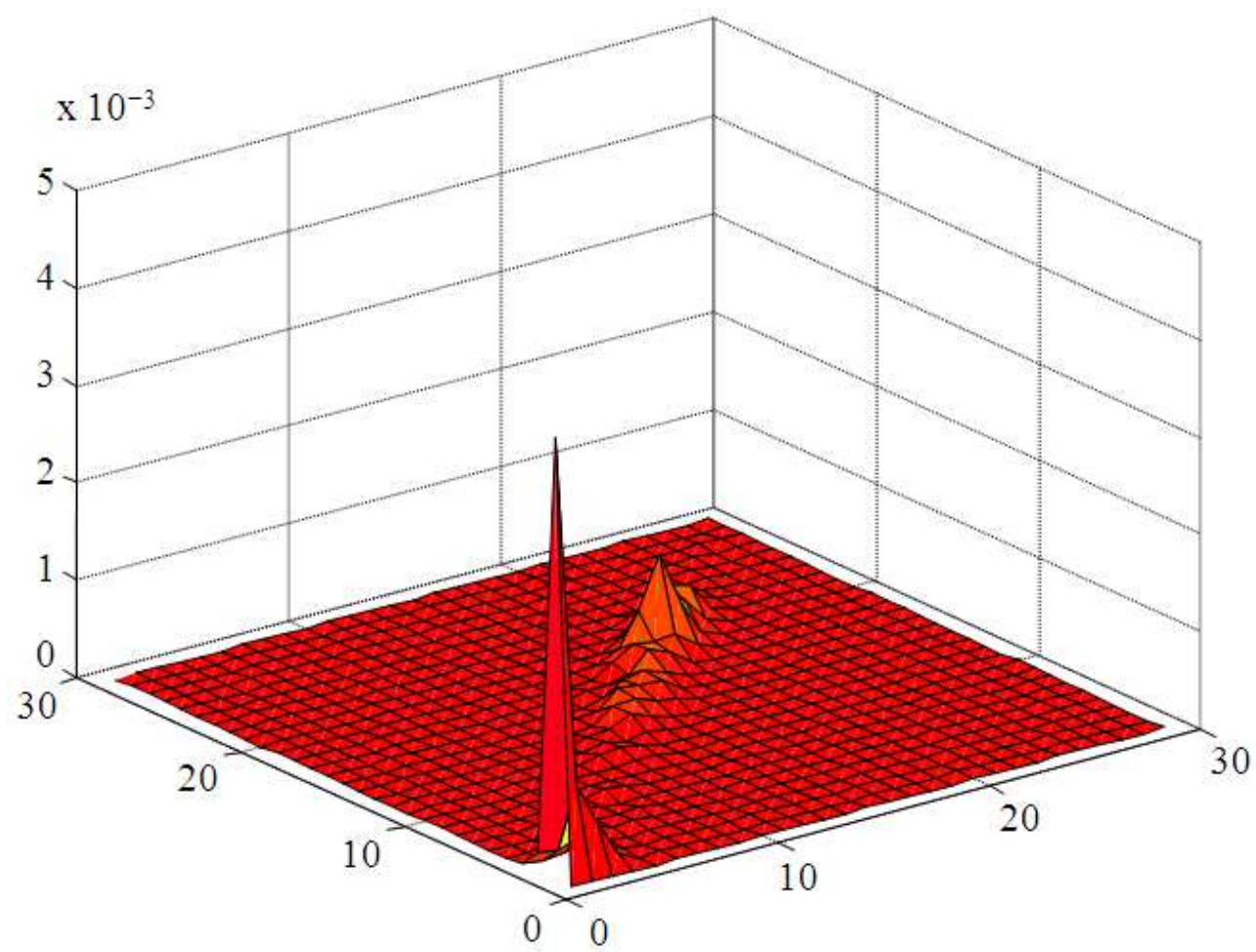

(e) 

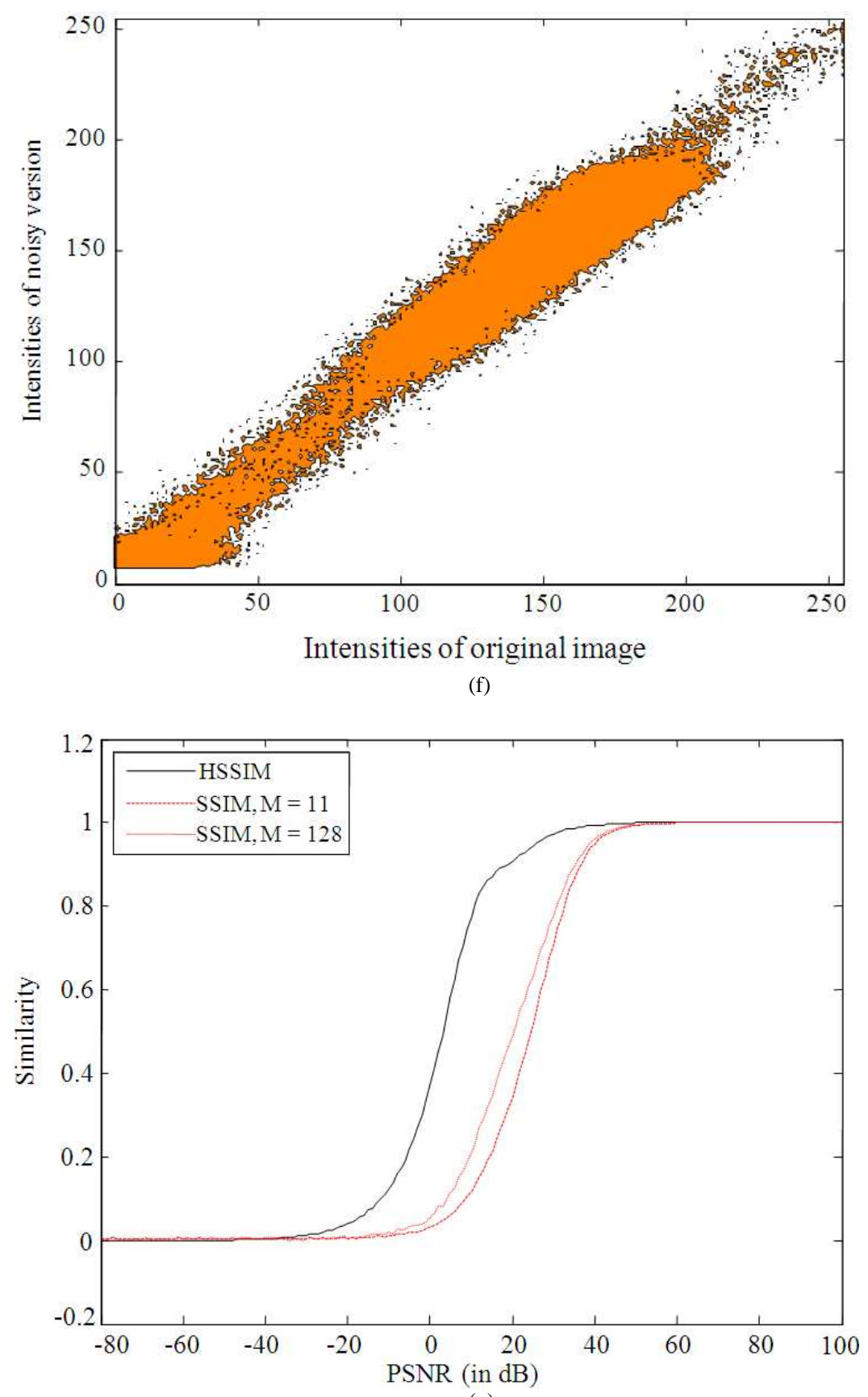

(g)

Fig. 1. Performance of HSSIM and SSIM using an image of landscape under Gaussian noise, (a) The test images (MATLAB Cameraman), (b) Histograms of original and noisy images, (c) Joint histogram of identical images (noise-free), (d) Contour plots for joint histogram of identical images (noise-free): Almost a straight line around $\mathrm{i}=\mathrm{j}$, (e, f) Joint histogram and its contour of original and noisy images. Symmetry can be detected around $\mathrm{i}=\mathrm{j}$, (g) Similarity vs. PSNR (dB): It is clear that HSSIM outperforms the conventional SSIM in its capability to detect similarity at low PSNR (almost $20 \mathrm{~dB}$ difference). Note that increasing the processing window length $\mathrm{M}$ up to half image width can improve SSIM performance a bit (dotted curve); however, further increase of M could give messy results 
Asmhan Flieh Hassan et al. / Journal of Computer Science 10 (11): 2269.2283, 2014
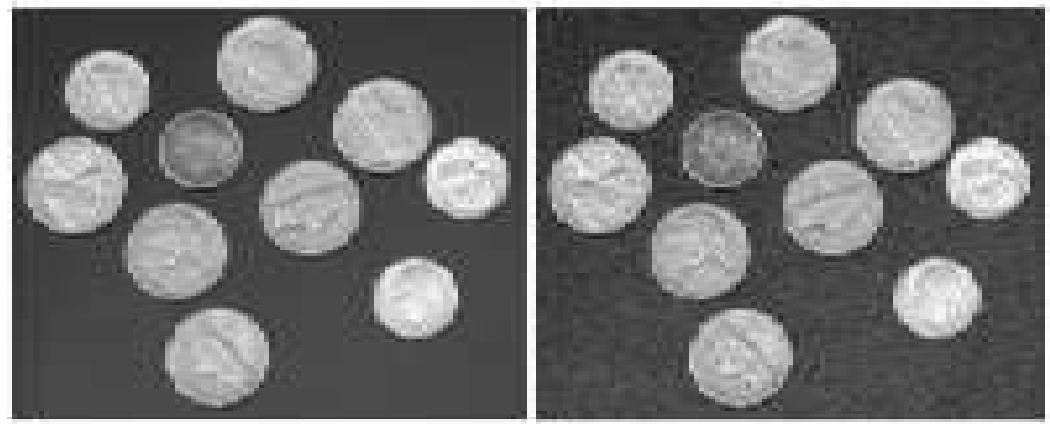

(a)

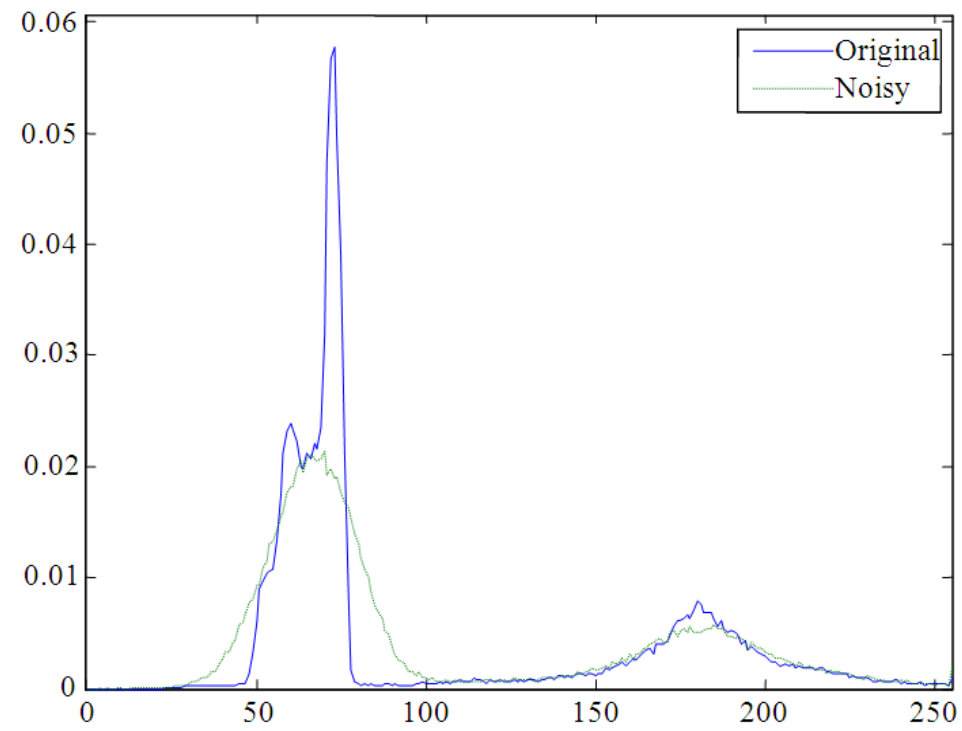

(b)

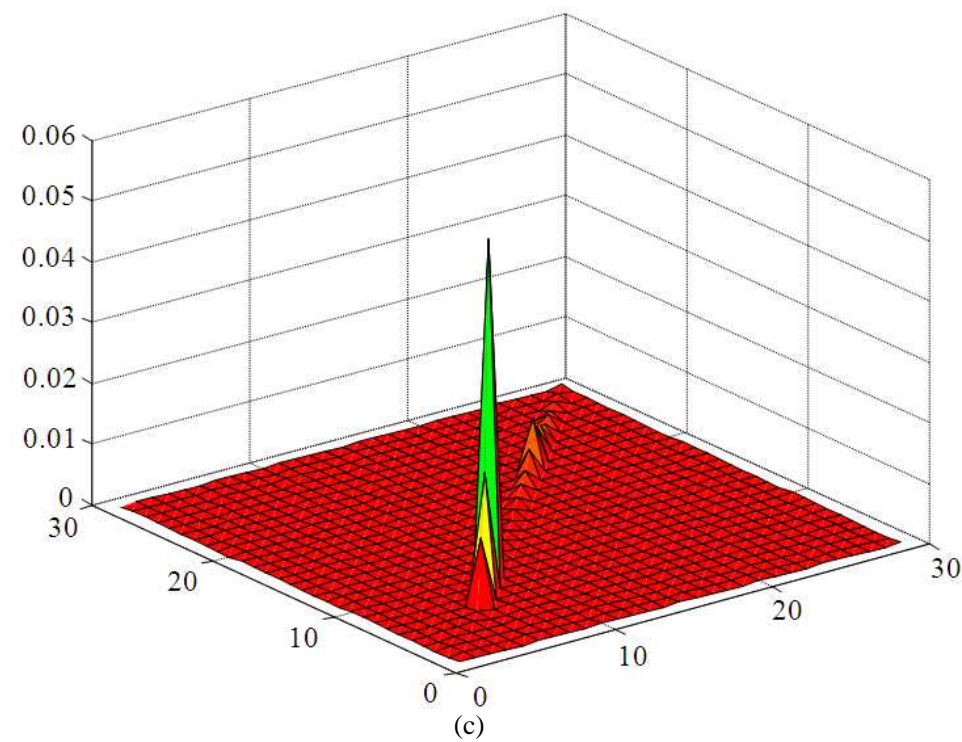

2276 
Asmhan Flieh Hassan et al. / Journal of Computer Science 10 (11): 2269.2283, 2014

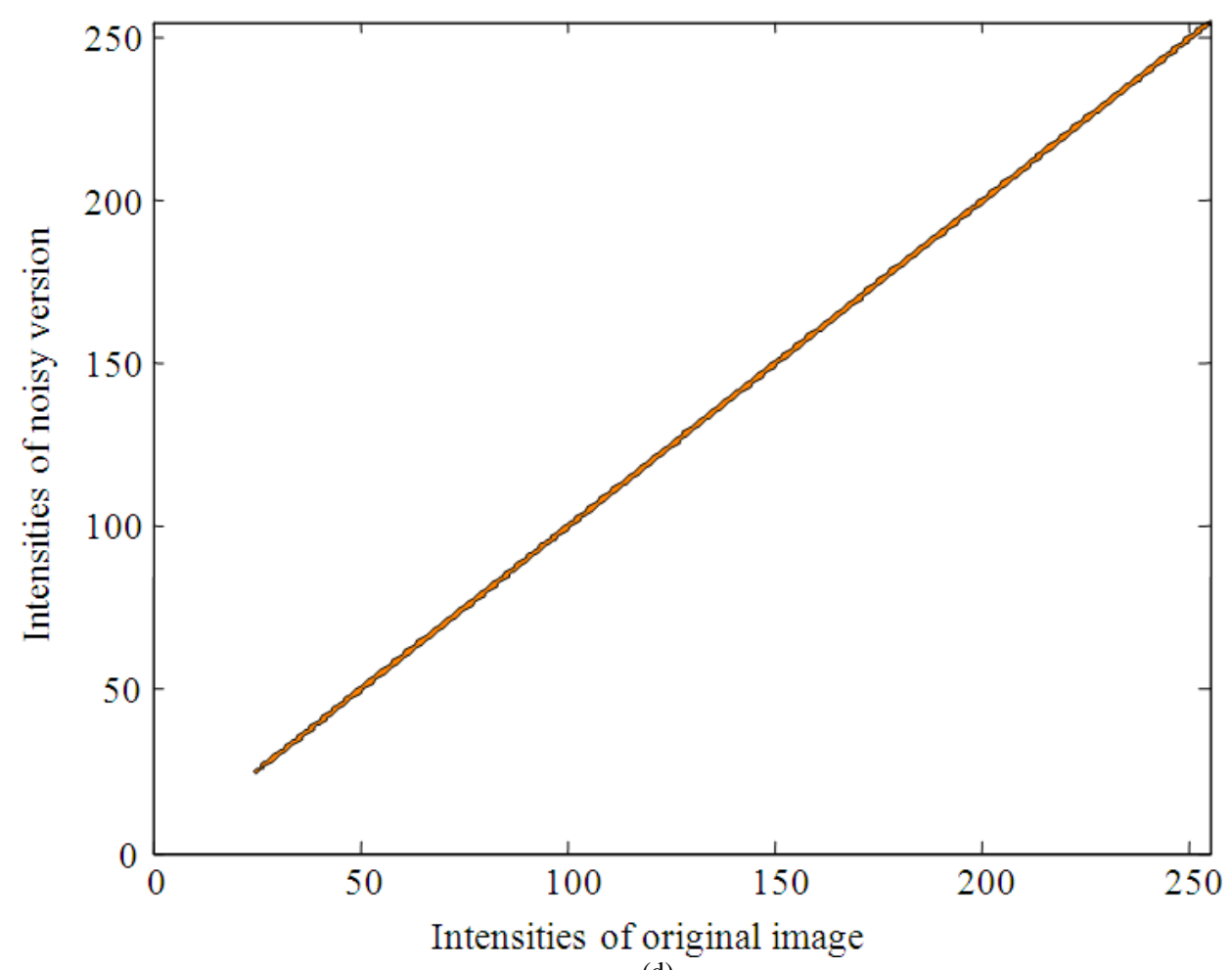

(d)

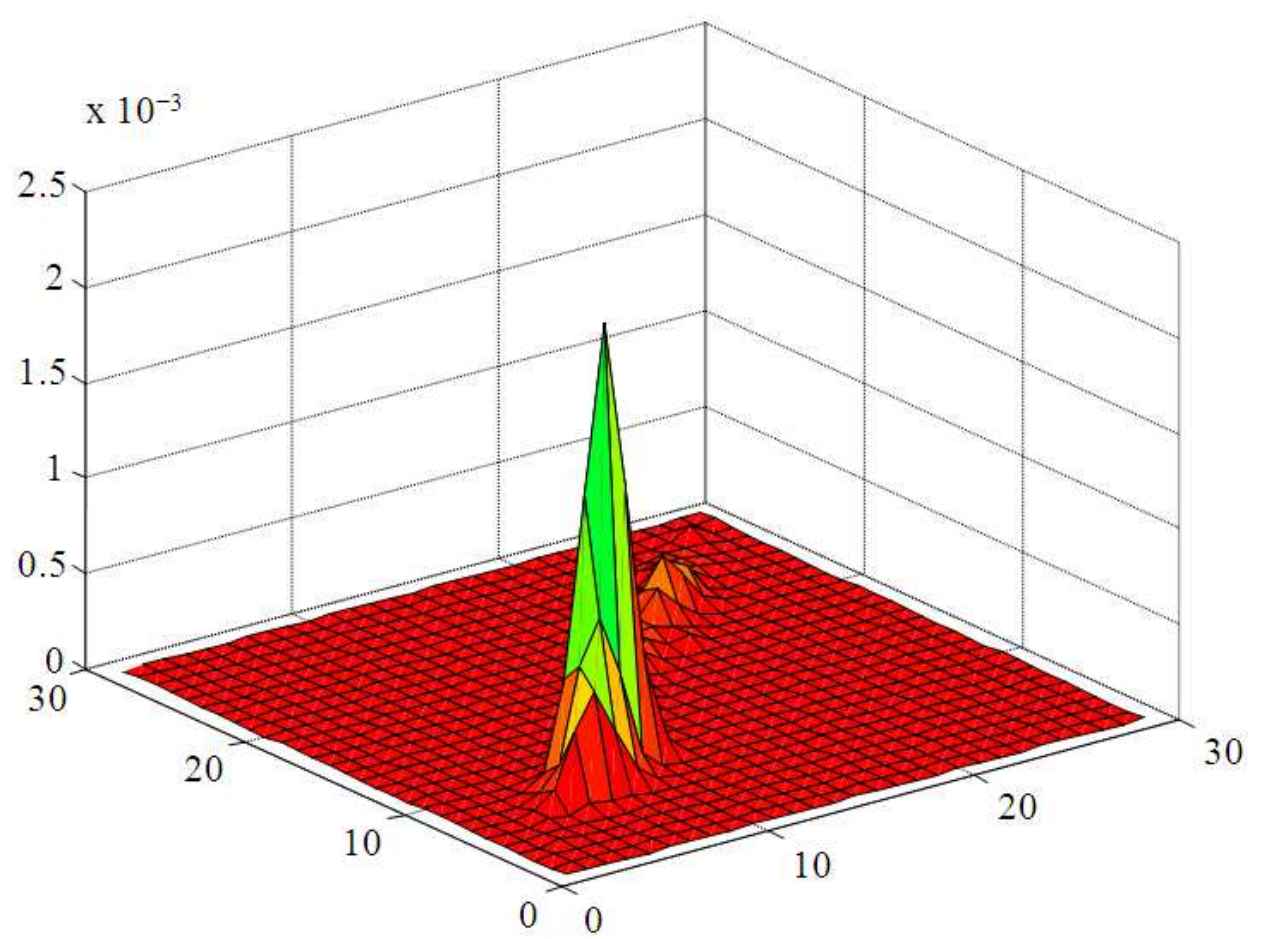

(e) 


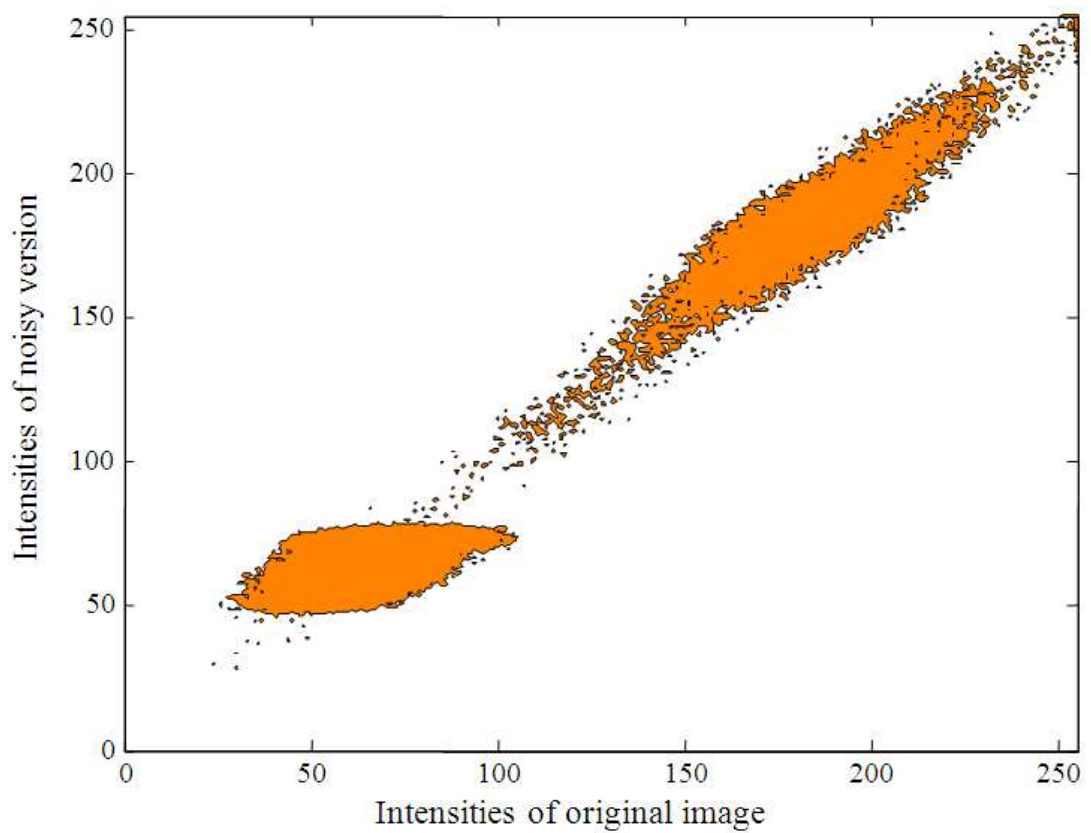

(f)

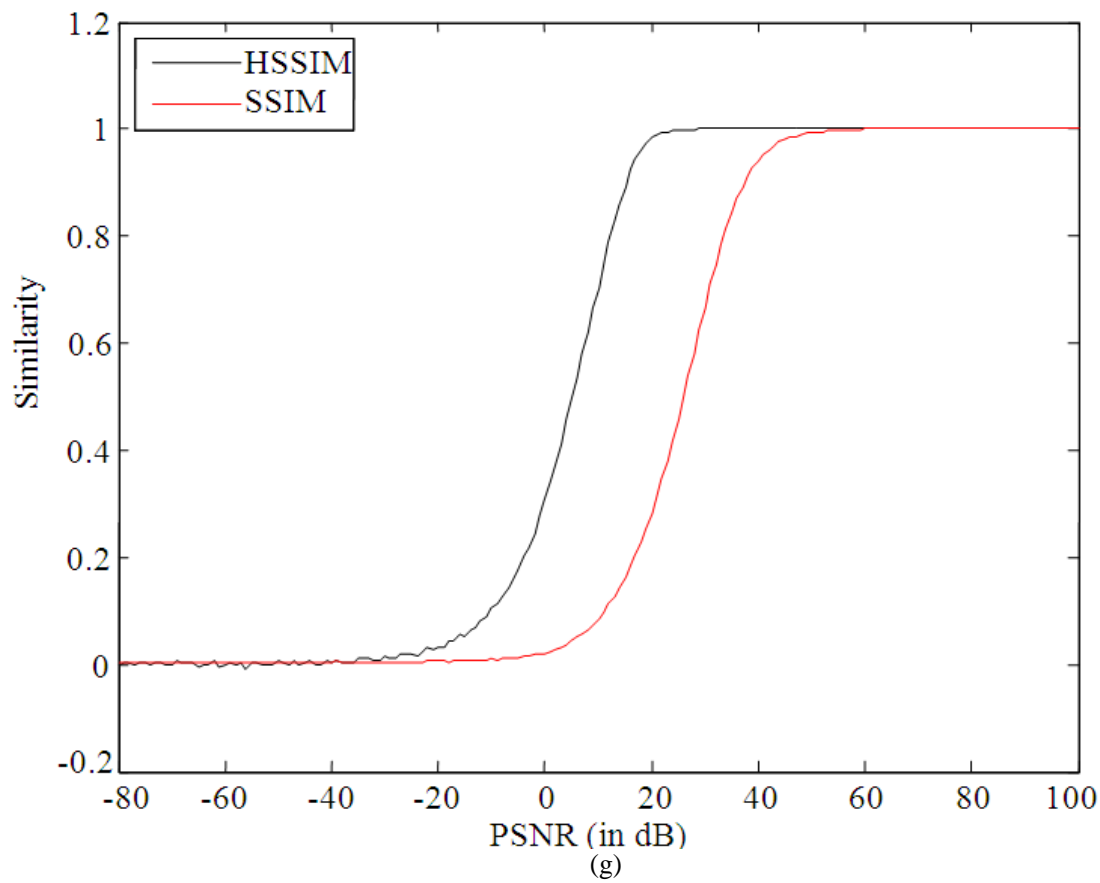

Fig. 2. Performance of HSSIM and SSIM $(M=11)$ for geometric images (taken from MATLAB). Note that HSSIM still outperforms the conventional SSIM in its capability to detect similarity at low PSNR (almost $20 \mathrm{~dB}$ difference). Compare with Figure 1, (a) Original Image, Noisy Image, PSNR $(d B)=28$, (b) Histograms: Original solid; noisy dotted, PSNR $(\mathrm{dB})=$ 28, (c) Joint Histogram: Identical images, PSNR (dB) = infinity, (d) Jt. Hist. (Contour): Identical images, PSNR $(\mathrm{dB})=$ infinity, (e) Joint Histogram: Original image and noisy version, PSNR $(\mathrm{dB})=28$, (f) Jt. Hist. (Contour): Original and noisy image, PSNR $(\mathrm{dB})=28,(\mathrm{~g})$ Similarity vs. PSNR $(\mathrm{dB})$ 
Asmhan Flieh Hassan et al. / Journal of Computer Science 10 (11): 2269.2283, 2014
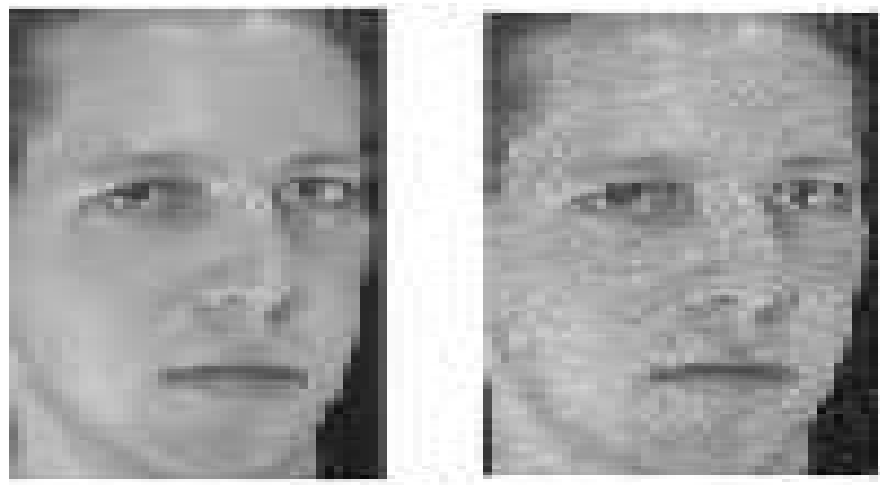

(a)

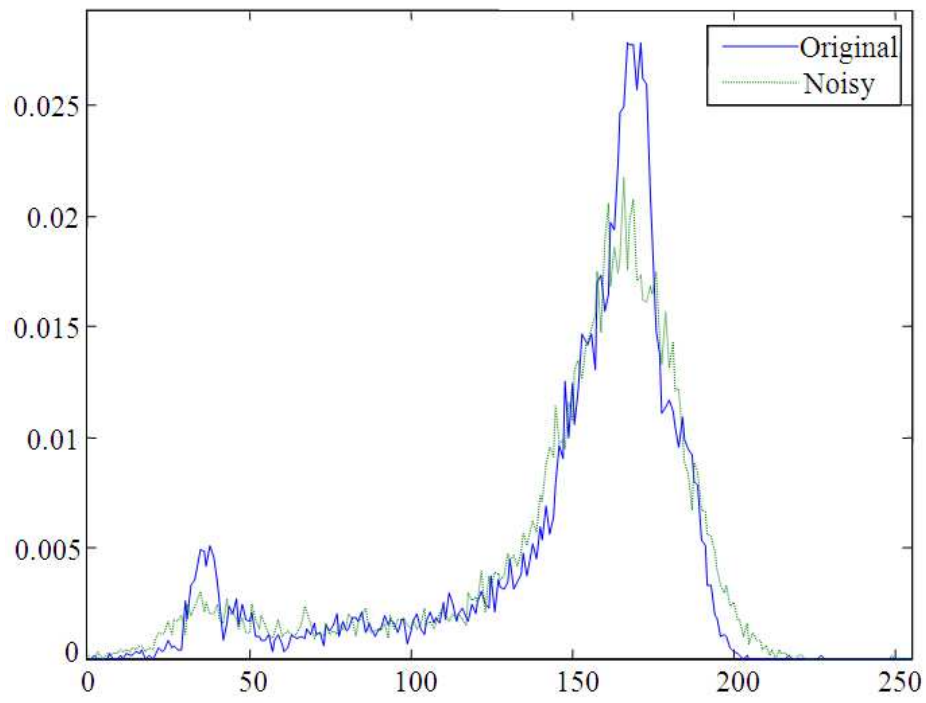

(b)

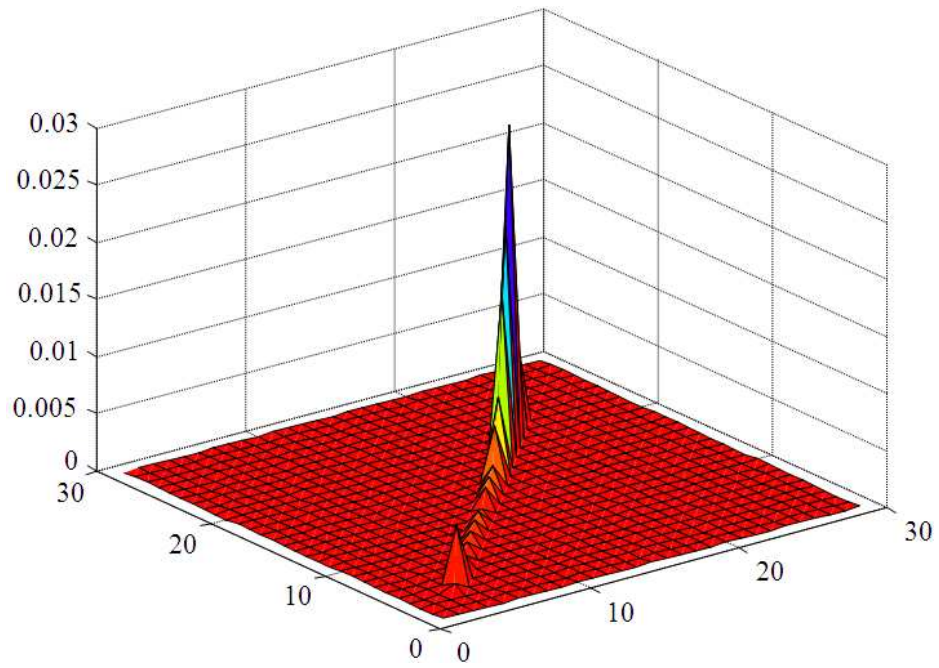

(c)

Science Publications 
Asmhan Flieh Hassan et al. / Journal of Computer Science 10 (11): 2269.2283, 2014

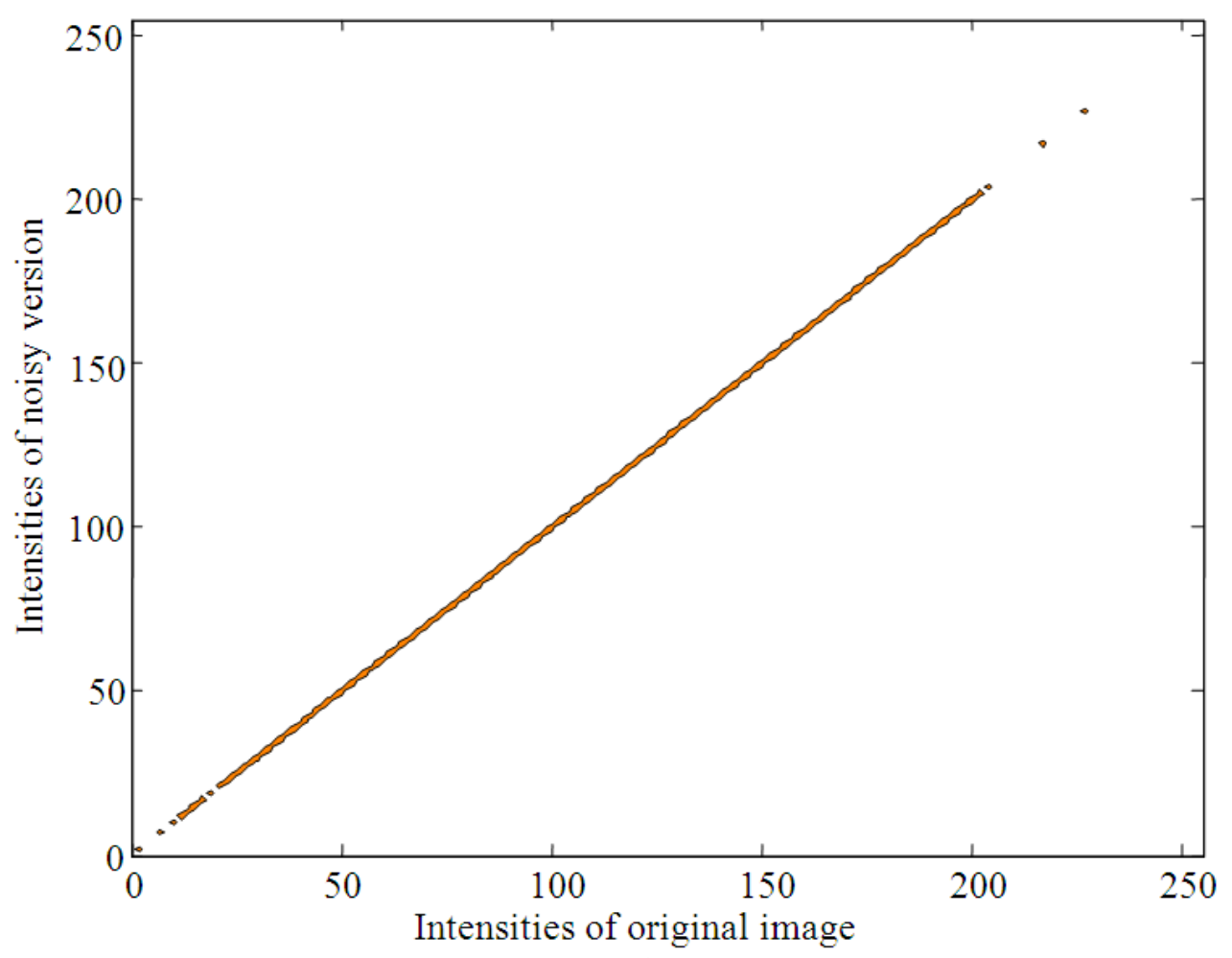

(d)

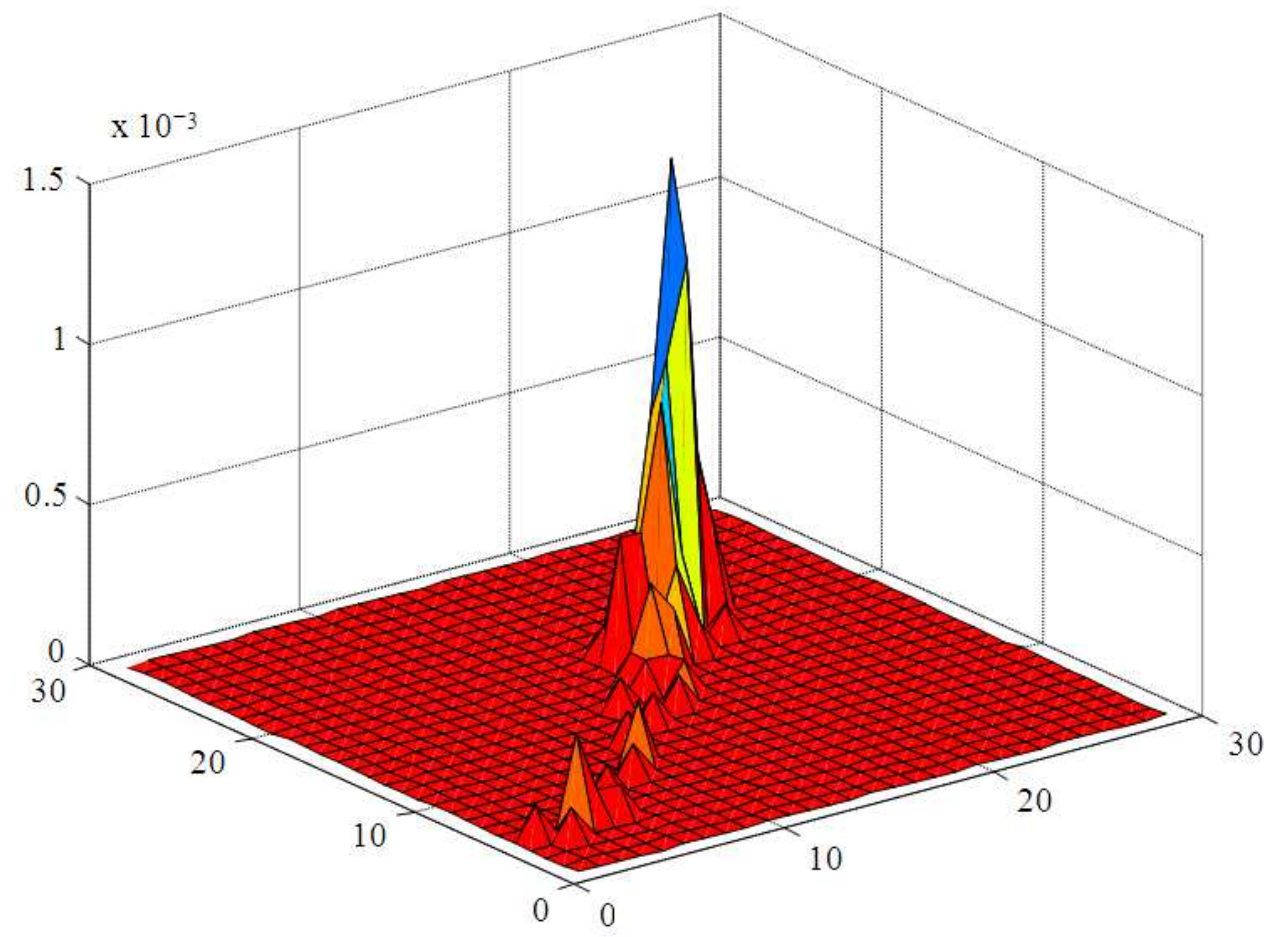

(e) 


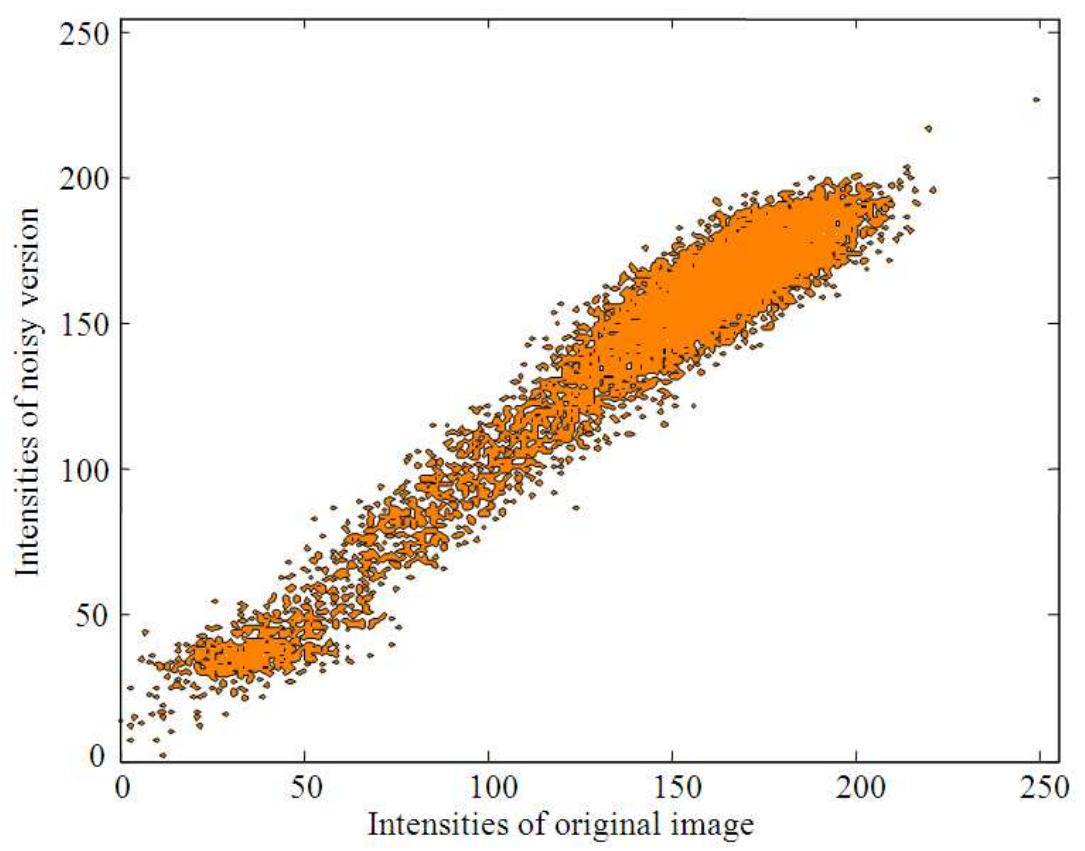

(f)

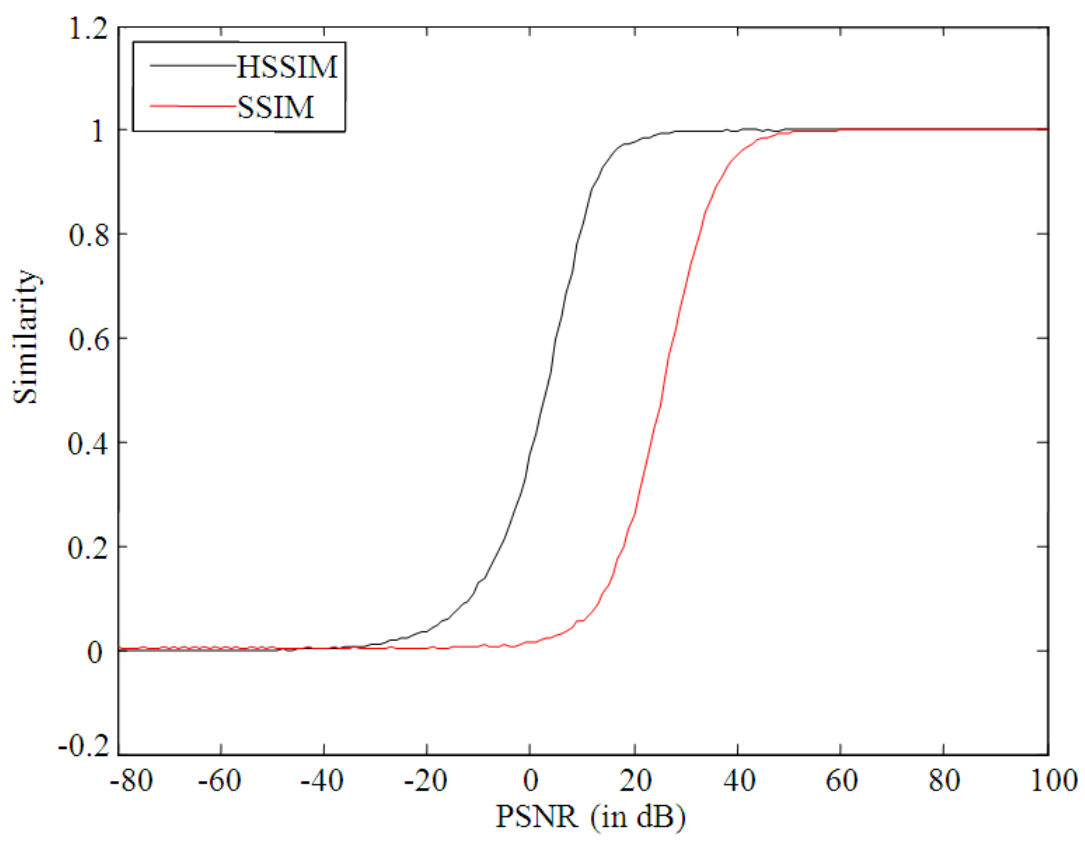

(g)

Fig. 3. Performance of HSSIM and SSIM $(M=11)$ for a human face (taken from AT and T Database). Note that HSSIM still outperforms the conventional SSIM in its capability to detect similarity at low PSNR (almost $20 \mathrm{~dB}$ difference; comparable with Figures 1 and 2), (a) Original Image, Noisy Image, PSNR $(\mathrm{dB})=28$, (b) Histograms: Original solid; noisy dotted, PSNR $(\mathrm{dB})=28$, (c) Joint Histogram: Identical images, PSNR (dB) = infinity, (d) Jt. Hist. (Contour): Identical images, PSNR $(\mathrm{dB})=$ infinity, (e) Joint Histogram: Original image and noisy version, PSNR $(\mathrm{dB})=28$, (f) Jt. Hist. (Contour): Original and noisy image, PSNR $(\mathrm{dB})=28,(\mathrm{~g})$ Similarity Vs. PSNR $(\mathrm{dB})$ 
We used the images "cameraman" and "coins" from MATLAB and a human face from AT and $\mathrm{T}$ face database (DOF, 1992).

The performance of HSSIM as compared to SSIM (represented by Equation 1) is shown in Fig. 1 to 3, which include a test under Gaussian noise. These results show about $20 \mathrm{~dB}$ difference of capability for HSSIM over SSIM to detect similarity at low PSNR.

\subsection{Effects of Analysis Parameters}

We noticed that the parameter $\mathrm{c}$ in the proposed error estimate (as per Equation 1) has no significant effect as long as it is kept small. Also, the choice of the window length $M$ has no significant effect as long as it is kept at reasonable values for local treatment as per (Wang and Bovik, 2004). Choosing a larger window length can improve to some extent the SSIM performance as shown in Fig. 1, where $M$ is varied up to half the image width. This is due to the fact that increasing the analysis window length may add more correlative information. However, increasing $\mathrm{M}$ beyond that value may result in degrading SSIM performance, where it gives messy result for $\mathrm{M}=$ image width.

\section{CONCLUSION}

A novel information-theoretic, image-dependent quality assessment measure has been proposed and tested versus structural similarity measure (SSIM) under Gaussian noise. The measure is based on joint histogram. Note that no interpolation has been used in this paper. Using interpolation can further improve performance, however, it would be the focus of a future work. It is shown that the proposed measure HSSIM out performs the conventional SSIM by almost $20 \mathrm{~dB}$ difference of PSNR in noisy environments for various kinds of images: Landscape, human face and geometric images. Increasing the window length of SSIM can improve its performance a bit; however, a significant increase may degrade its performance.

\section{ACKNOWLEDGEMENT}

The researchers would like to thank Hubei Provincial Key Laboratory of Mathematical Physics for financial support. Many thanks to the Reviewers for their constructive comments.

\section{ADDITIONAL INFORMATION}

\subsection{Funding Information:}

This research is funded by the Ministry of Higher Education and Scientific Research (Iraq) and supported by HuaZhong Normal University (China).

\subsection{Author's Contributions}

Asmhan Flieh Hassan: She is the main contributor, as this paper is part of her $\mathrm{PhD}$ program at the School of Mathematics and Statistics, HuaZhong Normal University, China. She contributed to preparation, analysis and algorithmic simulation.

Dong Cai-lin: He is the First Supervisor from School of Mathematics and Statistics, HuaZhong Normal University, China. He contributed to the preparation, mathematical analysis, and review of the paper.

Zahir M. Hussain: $\mathrm{He}$ is the Second Supervisor, from the Faculty of Computer Science and Mathematics, University of Kufa, Najaf, Iraq; also a Professor (adjunct) at the School of Engineering, Edith Cowan University, Australia. He contributed to preparation, analysis, computer simulations, and review of the paper.

\subsection{Ethics:}

The Authors utilized the standard AT\&T face database in this research, which is an internationally acknowledged database. All analyses and simulations in this work have been performed by the Authors. To the best of the Author's knowledge, this work is clear of any ethical issues.

\section{REFERENCES}

Darkner, S. and J. Sporring, 2013. Locally orderless registration. IEEE Trans. Patt. Anal. Machine Intell., 35: 1437-1450. DOI: 10.1109/TPAMI.2012.238

DOF, 1992. AT and T face database. The Database of Faces.

Hashim, A.N. and Z.M. Hussain, 2014. Novel imagedependent quality assessment measures. J. Comput. Sci., 2014: 1548-1560. DOI: 10.3844/jcssp.2014.1548.1560

Hill, D.L.G., D.J. Hawkes, N.A. Harrison and C.F. Ruff, 1993. A strategy for automated multimodality image registration incorporating anatomical knowledge and imager characteristics. In: Information Processing in Medical Imaging, Barrett, H.H. and A.F. Gmitro (Eds.), Springer Berlin Heidelberg, ISBN-10: 978-3540-56800-1, pp: 182-196. 
Klein, S., J.P.W. Pluim, M. Staring and M. Viergever, 2009. Adaptive stochastic gradient descent optimisation for image registration. Int. J. Comput. Vis., 81: 227-239. DOI: 10.1007/s 11263-008-0168-y

Klein, S., M. Staring and J. Pluim, 2007. Evaluation of optimization methods for nonrigid medical image registration using mutual information and b-splines. IEEE Trans. Imag. Process., 16: 2879-2890. DOI: 10.1109/TIP.2007.909412

Klein, S., U.A. van der Heide, I.M. Lips, M. van Vulpen and J.P.W. Pluim et al., 2008. Automatic segmentation of the prostate in 3D MR images by atlas matching using localized mutualinformation. Med. Phys., 35: 1407-1407. DOI: 10.1118/1.2842076

Kuczynski, K. and P. Miko£Ajczak, 2003. Information theory based medical images processing. OptoElect. Rev., 11: 253-259.

Loeckx, D., P. Slagmolen, F. Maes, D. Vandermeulen and P. Suetens et al., 2010. Nonrigid image registration using conditional mutual information. IEEE Trans. Med. Imag., 29: 19-29. DOI: 10.1109/TMI.2009.2021843

Maes, F., A. Collignon, D. Vandermeulen, G. Marchal and P. Suetens et al., 1997. Multimodality image registration by maximization of mutual information. IEEE Trans. Med. Imag., 16: 187-198. DOI: $10.1109 / 42.563664$

Pass, G. and R. Zabih, 1999. Comparing images using joint histograms. Multimed. Syst., 7: 234-240. DOI: 10.1007/s005300050125

Pluim, J.P.W., J.B.A. Maintz and M.A. Viergever, 2003. Mutual-information-based registration of medical images: A survey. IEEE Trans. Med. Images, 22: 986-1004. DOI: 10.1109/TMI.2003.815867

Sampat, M.P., Z. Wang, S. Gupta, A.C. Bovik and M.K. Markey et al., 2009. Complex wavelet structural similarity: A new image similarity index. IEEE Trans. Image Process., 18: 2385-2401. DOI: 10.1109/TIP.2009.2025923

Shannon, C.E. and W. Weaver, 1967. Mathematical Theory of Communication. 11th Edn., University of Illinois Press, Urbana, pp: 125.

Sheikh, H.R., M.F. Sabir and A.C. Bovik, 2006. A statistical evaluation of recent full reference image quality assessment algorithms. IEEE Trans. Image Process., 15: 3440-3451. DOI: 10.1109/TIP.2006.881959
Simard, P.Y., Y. Le Cun, J.S. Denker and B. Victorri, 2000. Transformation invariance in pattern recognition: Tangent distance and propagation. Int. J. Imag. Syst. Technol., 11: 181-197. DOI: 10.1002/1098-1098(2000)11:3<181::AIDIMA1003>3.0.CO;2-E

Studholme, C., C. Drapaca, B. Iordanova and V. Cardenas, 2006. Deformation-based mapping of volume change from serial brain mri in the presence of local tissue contrast change. IEEE Trans. Med. Imag., 25: 626-639. DOI: 10.1109/TMI.2006.872745

Studholme, C., D. Hill and D. Hawkes, 1999. An overlap invariant entropy measure of 3D medical image alignment. Pattern Recognit., 32: 71-86. DOI: 10.1016/S0031-3203(98)00091-0

Viola, P. and W.M.I.I.I. Wells, 1995. Alignment by maximization of mutual information. Proceedings of the 5th International Conference on Computer Vision, Jun. 20-23, IEEE Xplore Press, Cambridge, MA, pp: 16-23. DOI: 10.1109/ICCV.1995.466930

Wang, Z. and A.C. Bovik, 2004. A universal Image quality index. IEEE. Signal Process. Lett., 9: 81-84. DOI: $10.1109 / 97.995823$

Wang, Z., A.C. Bovik and L. Lu, 2002. Why is image quality assessment so difficult. Proceedings of the IEEE International Conference on Acoustics, Speech and Signal Processing, May 13-17, IEEE Xplore Press, Orlando, FL, USA, pp: 3313-3316. DOI: 10.1109/ICASSP.2002.5745362

Wang, Z., A.C. Bovik, H.R. Sheikh and E.P. Simoncelli, 2004. Image quality assessment: From error visibility to structural similarity. IEEE Trans. Image Process., 13: 600-612. DOI: 10.1109/TIP.2003.819861

Wells, W.M., P. Viola, H. Atsumi, S. Nakajima and R. Kikinis, 1996. Multi-modal volume registration by maximization of mutual information. Med. Image Anal., 1: 35-51. PMID: 9873920.

Zhang, F., S. Li, L. Ma and K.N. Ngan, 2009. Limitation and challenges of image quality measurement. Visual Commun. Image Process. DOI: $10.1117 / 12.863083$

Zhuang, S., D. Arridge, D. Hawkes and S. Ourselin, 2011. A nonrigid registration framework using spatially encoded mutual information and freeform deformations. IEEE Trans. Med. Imag., 30: 1819-1828. DOI: 10.1109/TMI.2011.2150240 